\title{
FASTPERIODISCHE LÖSUNGEN DER WELLENGLEICHUNG.
}

\section{VoN}

\section{S. BOCHNER}

in Princeton N. J.

Wir meinen die Gleichung

$$
\sum_{p=1}^{k} \frac{\partial}{\partial x_{p}}\left(\sum_{q=1}^{k} a_{p q}(x) \frac{\partial \varphi}{\partial x_{q}}\right)=\mu(x) \frac{\partial^{2} \varphi}{\partial t^{2}} \quad a_{p q}(x)=a_{\eta p}(x)
$$

mit »elliptischer» linker Seite [Präzisierung erfolgt später], welche eine Verallgemeinerung der klassischen Wellengleichung

$$
\sum_{p=1}^{k} \frac{\partial^{2} \varphi}{\partial x_{p}^{2}}=\mu(x) \frac{\partial^{2} \varphi}{\partial t^{2}}
$$

auf krummlinige Koordinaten bzw. Räume ist. Die Koeffizienten $a_{p q}, \mu$ sind Funktionen der $x_{1}, \ldots, x_{k}$ in einem beliebigen (auch unbeschränkten) offenen Gebiet $G$ des $k$-dimensionalen Raumes, und auch alle sonstigen Funktionen der $x$ werden nur in diesem offenen Gebiet betrachtet werden. Wenn eine Funktion, wie z. B. eine Lösung $\varphi(x, t)$ von (I), auch von $t$ abhängt, so durchläuft $t$ das Gesamtintervall $[-\infty, \infty]$.

U̇blicherweise werden die Lösungen von (I) dahin eingeschränkt, dass sie für jedes $t$ als Funktionen in $x$ gewissen "Randbedingungen" genügen sollen, z. B. dass sie verschwindende Randwerte haben. Wie auch diese Randbedingungen im Einzelnen beschaffen sein mögen, sie sind immer »linear». D. h. bezeichnet man mit $\mathfrak{F}$ die Gesamtheit der Funktionen in $x$ mit diesen Randbedingungen, und ist $f_{1} \subset \mathfrak{F}, f_{2} \subset \mathfrak{F}$, und sind $c_{1}, c_{2}$ beliebige Zahlen, so ist auch $c_{1} f_{1}+c_{2} f_{2} \subset F^{1}$

${ }^{1}$ Alle Zahlen und Funktionswerte sind bis auf weiteres reell. 
Für die meisten, wenn auch nicht alle, Arten von Randbedingungen geniigen die Lösungen von (I) dem Energieprinzip, welches besagt, dass das Integral

$$
E=\frac{\mathbf{I}}{2} \int_{G}\left(\sum_{p, q=1}^{k} a_{p q} \frac{\partial \varphi}{\partial x_{p}} \frac{\partial \varphi}{\partial x_{q}}+\mu\left(\frac{\partial \varphi}{\partial t}\right)^{2}\right) d x
$$

von $t$ unabhängig ist. Auf die Gültigkeitsfrage kommen wir später zurück. Neuerdings hat Herr Muckenhoupt ${ }^{1}$, im Falle $k=\mathrm{I}$, und für die einfachsten Randbedingungen eine wichtige Beziehung zwischen dem Energiesatz und dem zeitlichen Verlauf der Lösungen aufgewiesen. Er hat gezeigt, dass, "im quadratischen Mittel über $x$, die Funktion $\varphi(x, t)$ eine fastperiodische Funktion von $t$ ist. Dieses Resultat wollen wir möglichst allgemein und unter möglichst scharfer Herausstellung der zugrundeliegenden Voraussetzungen erneut begründen. Die Begründung wird, wie bei Muckenhoupt, in einer direkten Verifikation der Bohrschen Definition der fastperiodischen Funktionen durch relativ dichte [arithmetisch nicht spezifizierte] Verschiebungszahlen bestehen. Die Schwingungseigenschaften der fastperiodischen Funktionen scheinen in dieser wichtigen Anwendung, ebenso wie im Bohrschen Aufbau der Theorie, tatsächlich den Verschiebungseigenschaften im Range nachzustehen; hierüber wolle sich der Leser aus dem Gang der nachfolgenden Betrachtung selber ein Bild machen.

Das ganze Resultat ist überraschend, wenn man sich seine physikalische Deutung vergegenwärtigt. Fis besagt, dass im Falle eines beliebig krummlinigen Raumes, sofern nur die Zeitkoordinate unrelativistisch abgesondert ist, jede energie-konstante Welle in einem fastperiodischen Rhythmus schwingt.

\section{$\S$ I. Der Kuergiesatz.}

Zur eigentlichen Fastperiodizitätsaussage sind verschiedene Voraussetzungen notwendig, die wir im Kursiv-druck hervorheben und fortlaufend mit römisehen Zahlen numerieren werden. Wir bemerken, dass man verschiedene unter den Voraussetzungen mildern, zusammenziehen, oder gänzlich auseinander ableiten könnte. Aber wir wollen uns absichtlich von solchen akzessorischen Betrachtungen möglichst freihalten.

${ }^{1}$ C. F. Muckenhoupt, Almost periodic functions and vibrating systems. Journal of Mathematics and Physics, Massachusetts Institute of Technology, 8 (1929), I63-I98. 
I. The Funktion $\mu(x)$ ist stetig, und die Funktionen $a_{p q}(x)$ besitzen durchweg stetige partielle Ableitungen bis zur zweiten Ordnung.

II. Vorgegeben ist eine lineare Klasse $\mathfrak{F}$ von Funktionen in $x$, von denen jede stetige partielle Ableitungen bis zur zweiten Ordnung besitzt.

III. Für je zwei Funktionen $f, g$ aus $\mathfrak{F}$ existiert in irgend einem Sinne das Integral

$$
\int_{G}\left(\sum_{p, q} a_{p q} \frac{\partial f}{\partial x_{p}} \frac{\partial g}{\partial x_{q}}+f \sum_{p} \frac{\partial}{\partial x_{p}}\left(\sum_{q} a_{p q} \frac{\partial g}{\partial x_{q}}\right)\right) d x \equiv \int_{G} \sum_{p} \frac{\partial}{\partial x_{p}}\left(f \sum_{q} a_{p q} \frac{\partial g}{\partial x_{q}}\right) d x
$$

und hat den Wert null.

Diese Forderung des Nullwerdens läuft auf eine Randbedingung hinaus, wie man erkennt, wenn man im Falle eines "anständig" berandeten Gebiets $G$ das Integral rechts in ein Integral über die Berandung von $G$ verwandelt.

IV. Die Funktion $\varphi(x, t)$ ist eine festgehaltene Lösung von (I), von der Art, dass für jedes $t$ die Funktionen

$$
\varphi(x, t), \quad \frac{\partial}{\partial t} \varphi(x, t)
$$

$z u \mathfrak{F}$ gehören.

V. Das Integral (2) existiert, ist eine differenzierbare Funktion von $t$, und die Differentiation lässt sich unter dem Integral ausfïhren,

$$
\frac{d E}{d t}=\int_{G}\left(\sum_{p, q} a_{p q} \frac{\partial \varphi}{\partial x_{p}} \frac{\partial}{\partial x_{q}}\left(\frac{\partial \varphi}{\partial t}\right)+\frac{\partial \varphi}{\partial t} \cdot \mu \frac{\partial^{2} \varphi}{\partial t^{2}}\right) d x
$$

Wenn man hierin für $\mu \frac{\partial^{2} \varphi}{\partial t^{2}}$ die linke Seite von (I) einsetzt, und III. auf die Funktionen

$$
f \equiv \varphi, \quad g \equiv \frac{\partial \varphi}{\partial t}
$$

anwendet, so erhalten wir den

Energiesatz. Falls die Voraussetzungen I.--V. zutreffen, so ist

$$
E=\text { constans. }
$$

Um den Energiesatz anwenden zu können, benötigen wir weitere Voraussetzungen. 
VI. Jede Funktion $f$ aus $\mathfrak{F}$ ist mitsamt ihren ersten Ableitungen quadratisch integrierbar, d. $h$.

(4)

$$
\int_{G}\left(f^{2}+\left(\frac{\partial f}{\partial x_{1}}\right)^{2}+\cdots+\left(\frac{\partial f}{\partial x_{k}}\right)^{2}\right) d x<\infty
$$

und es gibt Zahle"

$$
0<c<C
$$

so dass

$$
\begin{gathered}
c \int_{G} \sum_{p}\left(\frac{\partial f}{\partial x_{p}}\right)^{2} d x \leqq \int_{G} \sum_{p, q} a_{p q} \frac{\partial f}{\partial x_{p} \partial x_{q}} d x \leqq C \int_{G} \sum_{p}\left(\frac{\partial f}{\partial x_{p}}\right)^{2} d x \\
c \int_{G} f^{2} d x \leqq \int_{G} u f^{2} d x \leqq C \int_{G} f^{2} d x
\end{gathered}
$$

wobei auch die zuei mittleren Integrale als existierend corausgesetzt werden.

Aus dem Energiesatz und VI. ergibt sich, wenn man die positive Zahl $A=\sqrt{\frac{C}{c}}$ einführt, die wichtige Relation

(5) $\underset{-\infty<t<\infty}{\text { Ob. Gr. }} \int_{G}\left(\sum_{p}\left(\frac{\partial \varphi}{\partial x_{p}}\right)^{2}+\left(\frac{\partial \varphi}{\partial t}\right)^{2}\right) d x \leqq A^{2} \cdot \underset{-x<t<\infty}{\operatorname{Unt}} \int_{G}\left(\sum_{p}\left(\frac{\partial \varphi}{\partial x_{p}}\right)^{2}+\left(\frac{\partial \varphi}{\partial t}\right)^{2}\right) d x$.

Die mit der Lösung $\varphi(x, t)$ für irgendein $\tau$ gebildete Funktion

$$
\varphi_{\tau}(x, t)=\varphi(x, t+\tau)-\varphi(x, t)
$$

ist wiederum eine Lösung, und wir setzen voraus:

VII. Die Voraussetzung V. wird von allen Funktionen

erfiillt.

$$
\varphi(x, t) \equiv \varphi_{\tau}(x, t) \quad(-\infty<\tau<\infty)
$$

Daraus folgt dann für die Funktion

(7) $\Phi\left(t_{1}, t_{2}\right)=\int_{G}\left[\sum_{p}\left(\frac{\partial \varphi\left(x, t_{1}\right)}{\partial x_{p}}-\frac{\partial \varphi\left(x, t_{2}\right)}{\partial x_{p}}\right)^{2}+\left(\frac{\partial \varphi\left(x, t_{1}\right)}{\partial t}-\frac{\partial y\left(x, t_{2}\right)}{\partial t}\right)^{2}\right] d x$

die Behauptung: 
Für alle $\tau$ ist

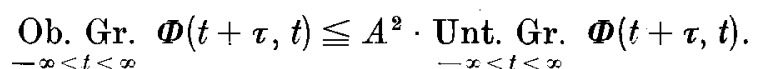

\section{$\S$ 2. Die Verschiebungseigenschaften.}

Eine (für alle $t$ ) definierte Funktion $F(t)$ heisse eine Muckenhouptsche Funktion, falls die Werte

$$
\alpha=F(t)
$$

nicht Zahlen, sondern quadratisch integrierbare Funktionen in $x$ sind. Wir ordnen jeder quadratisch integrierbaren Funktion $\alpha=f(x)$ als ihren "Betrag» $|\alpha|$ die Grösse

$$
\left[\int_{G} f^{2} d x\right]^{1 / 2}
$$

zu. In diesem Sinne nennen wir eine Muckenhouptsche Funktion $\boldsymbol{F}(t)$ /gleichmässig] stetig, falls

$$
\lim _{\tau \rightarrow 0}|F(t+\tau)-F(t)|=0
$$

[gleichmässig in $t$ ], und wir nennen sie fastperiodisch, falls, wörtlich wie bei Bohr, man zu jedem $\varepsilon$ ein $l$ angeben kann, so dass jede Umgebung $\left|\tau-\tau_{0}\right|<l$ eine Zahl $\tau=\tau(\varepsilon)$ enthält, für welche

$$
|F(t+x)-F(t)| \leqq \varepsilon \quad(-\infty<t<\infty)
$$

Wenn man die »abstrakte» Muckenhouptsche Funktion $F(t)$ als eine konkrete Funktion $F(x, t)$ ansieht, so besagt offenbar unsere Fastperiodizitätsdefinition, dass die letztere Funktion “im quadratischen Mittel über $x \gg$ fastperiodisch nach Bohr ist.

Die Muckenhouptschen Funktionen sind Spezialfälle allgemeiner »abstrakter» Funktionen, wie sie in einer voraufgehenden Arbeit des Verfassers ${ }^{1}$, die wir mit $\gg V \gg$ zitieren werden, systematisch untersucht wurden. Über solche allgemeinen Funktionen benötigen wir den folgenden

1 S. Bochner, Abstrakte fastperiodische Funktionen, Acta mathematica, 6I (I933), I49-1 84. 
Hilfssatz. Damit eine abstrakte Funktion $G(t)$ von der in $V », \S$ I betrachteten Art fastperiodisch (im dortigen Sinne) ist, sind die folgenden Voraussetzungen hinreichend:

I) $G(t)$ ist stetig und beschränkt,

2) der Wertevorrat von $G(t)$ ist kompakt, d. h. jede Folge $\left\{t_{n}\right\}$ enthält eine Teilfolge $\left\{t_{p_{n}}\right\}$, derart dass

$$
\lim _{m, n \rightarrow \infty}\left|G\left(t_{p_{m}}\right)-G\left(t_{p_{n}}\right)\right|=0
$$

3) es gibt eine Konstante $A>0$, so dass für alle $x$

$$
\underset{-\infty<t<x}{\text { Ob. Gr. }}|G(t+\tau)-G(t)| \leqq A \cdot \underset{\substack{\text { Unt. Gr.t<x } \\-x<t<x}}{ }|G(t+\tau)-G(t)|
$$

Beweis. Gegeben sei ein $\varepsilon>0$. Unter den Werten $\alpha=G(t)$ gibt es eine endliche Anzahl

$$
a_{1}, a_{2}, \ldots, \alpha_{n}
$$

derart, dass für alle $t$

$$
\operatorname{Min}_{v}\left|G(t)-\alpha_{v}\right| \leqq \varepsilon
$$

denn wenn dem nicht so wäre, so gä̈be es im Widerspruch zur Voraussetzung 2) eine unendliche Folge $\alpha_{n}=G\left(t_{n}\right)$, so dass für alle $m, n$

$$
\left|\kappa_{m}-\kappa_{n}\right|>\varepsilon
$$

Wir betrachten nun eine beliebige Funktion $H(t)$, welche nur die Werte (ro) annimmt, so dass

$$
|G(t)-H(t)| \leqq \varepsilon \quad(-\infty<t<\infty)
$$

und dass jeder Wert (Io) mindestens einmal angenommen wird. Wir betrachten nun irgendeine Umgebung

$$
|t|<l
$$

in welcher $H(t)$ jeden Wert mindestens einmal annimmt. Dann gibt es zu jedem $t_{1}$ ein $\tau_{1}$ aus der Umgebung (1 2 ), so dass

$$
\left|H\left(t_{1}\right)-H\left(\tau_{1}\right)\right|=0 .
$$


Nach (I I) ist

$$
\left|G\left(t_{1}\right)-G\left(\tau_{1}\right)\right| \leqq 2 \varepsilon
$$

und die Voraussetzung 3) ergibt für alle $t$

$$
\left|G\left(t+t_{1}-\tau_{1}\right)-G(t)\right| \leqq 2 A \varepsilon
$$

Die Zahl $t_{1}-\tau_{1}$ liegt aber in der Umgebung $\left|t-t_{1}\right|<l$, deren Mittelpunkt $t_{1}$ als beliebig angenommen wurde, und $l$ ist nur von $\varepsilon$ abhängig, womit der Hilfssatz bewiesen ist.

Wir kehren zu unserer Lösung $\varphi(x, t)$ zurück. Die Ableitungen

$$
\frac{\partial \varphi}{\partial x_{1}}, \ldots, \frac{\partial \varphi}{\partial x_{k}}, \frac{\partial \varphi}{\partial t}
$$

sind jede eine Muckenhouptsche Funktion in $t$, und auf Grund des Hilfssatzes wollen wir zeigen, dass sie fastperiodisch sind. Die unwesentlichen Voraussetzungen I) und 2) des Hilfssatzes müssen wir explizit formulieren, mit Ausnahme der Forderung der Beschränktheit, welche auf Grund von (5) von selbst erfüllt ist, also:

VIII. Jede der $k+I$ Funktionen (I3), als Muckenhouptsche Funktion $F(t)$ betrachtet,

I) ist stetig und

2) hat einen kompakten Wertevorrat, d.h. jede unendliche Folge $\left\{t_{n}\right\}$ enthält eine unendliche Teilfolge $\left\{t_{p_{n}}\right\}$, so dass

$$
\lim _{m, n \rightarrow \infty}\left|F\left(t_{p_{m}}\right)-F^{\prime}\left(t_{p_{n}}\right)\right|=0
$$

Die Voraussetzung 3) des Hilfssatzes hingegen ist mit der Relation (8) äquivalent. Sie folgt zwar nicht aus (8) für jede einzelne Funktion (13); wenn man aber die $k+$ I Funktionen (I3) als die vektoriellen Komponenten einer ab. strakten Funktion $G(t)$ auffasst, deren absoluter Betrag durch

$$
|G(t)|^{2}=\int_{G}\left(\sum_{p}\left(\frac{\partial \varphi}{\partial x_{p}}\right)^{2}+\left(\frac{\partial \varphi}{\partial t}\right)^{2}\right) d x
$$

definiert ist, so erfüllt letztere Funktion auf Grund von VIII. und (5) und (8) sämtliche Voraussetzungen des Hilfssatzes, und ist daher fastperiodisch. Wenn 30-33617. Acta mathematica. 62. Imprimé le 20 december 1933. 
$G(t)$ fastperiodisch ist so sind es auch die Komponenten (I3), und wir haben daher den

Satz 1. Falls die Voraussetzungen I.-VIII. erfïllt sind, so sind die $k+$ I Muckenhouptschen Funktionen (1 3) fastperiorlisch.

Insbesondere ist also die zeitliche Ableitung $\frac{\partial \varphi}{\partial t}$ von $\varphi(x, t)$ fastperiodisch. Was nun die Fastperiodizität von $\varphi(x, t)$ selber anbetrifft, so ist sie nicht mehr eine reine Folgerung aus dem Energiesatz. Um sie zu erzwingen, hat Muckenhoupt sich auf Probleme mit allereinfachsten Randbedingungen beschränken müssen. $\mathrm{Zu}$ einem allgemeinen Resultat gelangt man, wenn man ein allgemeines Kriterium darüber anwendet, wann das Integral einer (abstrakten) fastperiodischen Funktion wiederum fastperiodisch ist. Ein solches Kriterium ist in $\nabla », \$ 5$ gegeben worden, und aus den dortigen Betrachtungen ergibt sich, dass in unserem Falle die folgende zusätzliche Voraussetzung ausreicht.

IX. Die Muckenhouptsche Funktion $F(t) \equiv \varphi(x, t)$ ist beschränkt,

$$
|F(t)| \leqq \text { constans, }
$$

und ihr Werterorrat ist kompakt.

Und unser Satz lautet nun:

Satz 2. Falls die Voraussetzung IX. hinzugenommen wirl, so ist auch die Muckenhouptsche Funktion $\varphi(x, t)$ fastperiorlisch.

Die Voraussetzung (14) ist prinzipiell nicht ein Teil des Energieprinzips; aus dem Energieprinzip selber folgt eben nur die Fastperiodizität des "Wellenflusses» $\frac{\partial \varphi}{\partial t}$ und nicht der Welle selber. Und wir bemerken, dass die Betrachtungen des folgenden Paragraphen insofern von der Voraussetzung IX. unabhängig sind, als man in ihnen die Funktion $q(x, t)$ durch die Funktion $\frac{\partial \varphi(x, t)}{\partial t}$ ersetzen könnte, welche ja auch eine Iuösung von (I) ist, und deren Fastperiodizität von dieser Voraussetzung unabhängig ist.

\section{$\$$ 3. Die Schwingungseigenschaften.}

Wir wollen von jetzt an auch komplexwertige Lösungen von (I) zulassen [, die Koeffizienten $a_{p q}(x)$ und $\mu(x)$ sollen nach wie vor reell sein], und zwar wollen 
wir sowohl die Funktionenklasse $\mathfrak{F}$ als auch den Begriff der Lösung dahin erweitern, dass sie aus allen solchen Funktionen bestehen, deren Real- und Imaginärteil eine Funktion der bisherigen Klasse $\mathfrak{F}$ bzw. eine Lösung der bisher betrachteten Art ist. Als erstes machen wir die Voraussetzung:

X. Wenn man einem jeden Element $a=f(x)$ der (so erueiterten) Klasse $\mathfrak{F}$ den Betrag

$$
|\alpha|=\left[\int_{G}|f(x)|^{2} d x\right]^{1 / 2}
$$

zuordnet, so bildet $\mathfrak{F}$ einen komplex-linearen Raum gemäss der Definition in $V$, $\$ 3 \cdot 5$.

Nach " $V », \S 6$ kann man jeder fastperiodischen Funktion $F(t)$, deren Werte Elemente aus $\mathfrak{F}$ sind, eine sie eindeutig bestimmende Fourierreihe

$$
F(t) \sim \sum A_{n}(x) e^{i A_{n} t}
$$

zuordnen: die Exponenten sind gewisse reelle Zahlen, und die Koeffizienten $A_{n}(x)$ sind die durch

$$
A_{n}=\lim _{T \rightarrow \infty} \frac{\mathrm{I}}{2 T} \int_{-T}^{T} F(t) e^{-i A_{n} t} d t
$$

bestimmten Elemente aus $\mathfrak{F}$. Insbesondere setzen wir die Fourierreihe (I 5 ) für die Funktion

$$
F(t) \equiv \varphi(x, t)
$$

an, und wir wollen Bedingungen angeben, unter denen für je zwei zusammengehörige Grössen $\left(A_{n}, A_{n}\right)$ ihrer Fourierreihe die heuristisch sehr plausible Differentialgleichung

$$
\sum_{p} \frac{\partial}{\partial x_{p}}\left(\sum_{q} a_{p q} \frac{\partial A}{\partial x_{q}}\right)+\Lambda^{2} \mu A=\mathrm{o}
$$

besteht.

XI. Die partiellen Ableitungen

$$
\frac{\partial^{2} \varphi}{\partial x_{p} \partial x_{q}}
$$

sind in allen Punkten von $G$ vorhanden und stetig, und sind Elemente von $\mathfrak{F}$. 
XII. Von jeder der Funktionen

$$
f(x, t) \equiv \varphi, \frac{\partial \varphi}{\partial x_{1}}, \ldots, \frac{\partial \varphi}{\partial x_{k}}
$$

gilt folgendes. Wenn man sie durch die Werte uall z" ciner im ganzen k-dimensionalen Raum $R$ definierten Funktion erueitert, so gilt, für jedes $p$, von der »Differenz»

$$
D_{h}(t)=\int_{R}\left|\frac{f\left(x_{1}, \ldots, x_{p}+h, \ldots, x_{k} ; t\right)}{h}-\underline{f\left(x_{1}, \ldots, x_{p}, \ldots, x_{k} ; t\right)}-\frac{\partial f}{\partial x_{p}}\right|^{2} d x
$$

dass

$$
\lim _{h \rightarrow 0} D_{h}(t)=0 \quad \text { gleichmässig in } t \text {. }
$$

Auf Grund dieser zwei Voraussetzungen sind auch die Funktionen (17) fastperiodisch, und die Fourierreihen der Funktionen (I 7 ) und (I 8 ) entstehen aus der Fourierreihe von $\varphi(x, t)$ durch formale Differentiation. Um nun sicher zu sein, dass die beiden Seiten von (I) fastperiodisch sind, machen wir die Voraussetzungen :

XIII. Die Funktionen

$$
a_{p q}, \frac{\partial a_{p q}}{\partial x_{q}}, \frac{\partial^{2} a_{p q}}{\partial x_{\rho} \partial x_{\sigma}}, \mu
$$

rind beschränkt in $G$.

XIV. Die (abstrakte) Funktion $\frac{\partial^{2} \varphi}{\partial t^{2}}$ ist stetig in $t$.

Nunmehr sind wir fertig. Wenn man nämlich in ( 1 ) für die vorkommenden Ableitungen von $\varphi(x, t)$ die Fourierreihen einsetzt, und die Koeffizienten auf beiden Seiten vergleicht, so ergibt sich endgültig der

Satz 3. Falls die Voraussetzungen I.-XIV. erfiullt sind, so gilt von der Fourierreihe

$$
\varphi(x, t) \sim \sum A_{n}(x) e^{i \Delta_{n} t}
$$

dass jeder Bestandteil

$$
\varphi_{n}(x, t)=A_{n}(x) e^{i \Lambda_{n} t}
$$

gleichfalls eine denselben "Kandbedingungen» genügende Lösung von ( $\mathrm{I}$ ) ist. 
In welchem präzisen Sinne $\varphi(x, t)$ eine Summe der Bestandteile $\varphi_{n}(x, t)$ ist, wolle man aus $V », \S 6$ und $\$ 8$ entnehmen. Insbesondere ist die Parsevalsche Gleichung

$$
\sum_{n} \int_{G}\left|A_{n}(x)\right|^{2} d x=\lim _{T \rightarrow \infty} \frac{\mathrm{I}}{2} \int_{-T}^{T}\left[\int_{G}|\varphi(x, t)|^{2} d x\right] d t
$$

zu beachten.

29. Juli I933. 